\title{
Geometric Feature Estimators for Noisy Discrete Surfaces ${ }^{\star}$
}

\author{
L. Provot and I. Debled-Rennesson \\ LORIA Nancy \\ Campus Scientifique - BP 239 \\ 54506 Vandœuvre-lès-Nancy Cedex, FRANCE \\ $\{$ provot, debled\}@loria.fr
}

\begin{abstract}
We present in this paper robust geometric feature estimators on the border of a possibly noisy discrete object. We introduce the notion of patch centered at a point of this border. Thanks to a width parameter, attached to a patch, the noise on the border of the discrete object can be considered, and an extended flat neighborhood of a border point is computed. Stable geometric features are then extracted around this point. A normal vector estimator is proposed as well as a detector of convex and concave parts on the border of a discrete object.
\end{abstract}

\section{Introduction}

Geometric feature estimation on the surface of a discrete object is very important in digital geometry. For instance, in computer vision, rendering algorithms rely on the normal vector estimation on the surface of an object to produce a realistic view of this object. In image analysis, features such as area or curvature can be used for producing classification functions able to sort objects by shape or by size. In bioinformatics, in the protein-protein docking framework, one tries to associate two proteins according to their geometrical complementarity. Thus, it is important to locate critical areas on the surface, like holes (concave parts) and knobs (convex parts) or even big flat areas.

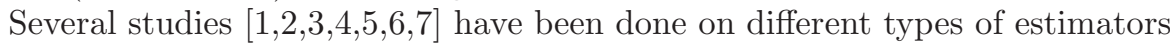
but the estimated geometric features are by definition very local. Thus, the noise introduced by acquisition tools like scanners or MRI, even weak, is disturbing for the estimator, and classical algorithms of digital geometry which rely on the regularity of discrete primitives do not always yield good results.

Therefore, for the last few years, the interest around the geometry of noisy objects has grown 891011. New discrete primitives such as blurred segments and blurred pieces of discrete planes have been introduced and, thanks to a width parameter, enable to take into account the noise in data while controlling the approximation. Encouraging results have been obtained in $2 \mathrm{D}$ for the curvature estimation, relying on blurred segments [12].

\footnotetext{
^ This work is supported by the ANR in the framework of the GEODIB project.
} 
In this paper we deal with the 3D case. In section 2, after recalling the definition of blurred pieces of discrete planes, we introduce the notion of a width- $\nu$ patch centered at $p$. In section 3, we then present some features (normal vector, area, outline) of a width $-\nu$ patch centered at $p$. With this material we develop, in section 4, an estimator able to distinguish between points that belong to concave, convex or flat parts of the surface of a possibly noisy discrete object. The paper ends up with some conclusions and perspectives in section 5 .

\section{Basic Notions and Definitions}

Hereafter we denote by $\mathcal{O}_{b}$ a possibly noisy 6-connected discrete object. We call surface or border of $\mathcal{O}_{b}$ the set of points $\mathcal{B}_{b}$ which have a 6 -neighbor that does not belong to $\mathcal{O}_{b}$.

Before presenting the notion of $a$ width- $\nu$ patch centered at $p$, we recall the definition of a width- $\nu$ blurred piece of discrete plane [11, which is the underlying discrete primitive of the patch.

\subsection{Blurred Pieces of Discrete Planes}

One can see a blurred piece of discrete plane as an arithmetic discrete plane for which some points are missing. More formally:

Definition 1. Let $N$ be a norm on $\mathbb{R}^{3}$ and $\mathcal{E}$ a set of points in $\mathbb{Z}^{3}$. We say that the discrete plane $\mathcal{P}(a, b, c, \mu, \omega)^{1}$ is a bounding plane of $\mathcal{E}$ if all the points of $\mathcal{E}$ belong to $\mathcal{P}$, and we call width of $\mathcal{P}(a, b, c, \mu, \omega)$, the value $\frac{\omega-1}{N(a, b, c)}$.

$A$ bounding plane of $\mathcal{E}$ is said optimal if its width is minimal.

Definition 2. A set $\mathcal{E}$ of points in $\mathbb{Z}^{3}$ is a width- $\nu$ blurred piece of discrete plane if and only if the width of its optimal bounding plane is less than or equal to $\nu$.

Two recognition algorithms of blurred pieces of discrete planes have been proposed in [11. The first one considers the Euclidean norm and, for a set of points $P$ in $\mathbb{Z}^{3}$, it solves the recognition problem by using the geometry of the convex hull of $P$. The second one considers the infinity norm and uses methods from linear programming to solve the recognition problem.

In the following sections, the results we present have been obtained by using the geometrical approach which uses the Euclidean norm.

\subsection{Width- $\nu$ Discrete Patches}

Here we are interested in the computation of different geometric features of a possibly noisy discrete surface, such as the normal vector, the area or the

\footnotetext{
${ }^{1}$ An arithmetic discrete plane $\mathcal{P}(a, b, c, \mu, \omega)$ is the set of integer points $(x, y, z)$ verifying $\mu \leq a x+b y+c z<\mu+\omega$, where $(a, b, c) \in \mathbb{Z}^{3}$ is the normal vector of the plane. $\mu \in \mathbb{Z}$ is named the translation constant and $\omega \in \mathbb{Z}$ the arithmetical thickness.
} 


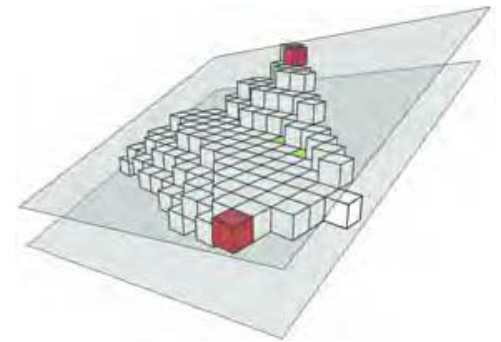

(a)

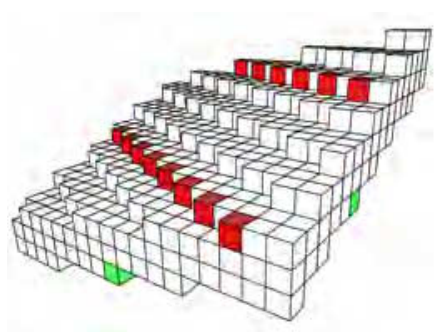

(b)

Fig. 1. (a) A width-3 blurred piece of discrete plane and (b) its optimal bounding plane $\mathcal{P}(4,8,19,-80,49)$, using the Euclidean norm

curvature. Numerous methods exist in the literature to compute such features in the discrete space. One can refer to [1132445] for normal vectors estimation and 37] for curvature estimators.

The problem is that these geometric features are by definition very local. And most of the defined estimators are very noise sensitive. But if we know that we are working on a noisy surface, we would like to use estimators that take into account the irregularity of this surface. A way to estimate these features at a point $p$ of the noisy surface is to use the information of the points lying in a neighborhood of $p$. The notion of patch we present hereafter takes place in this framework, considering an adaptive neighborhood around $p$.

Definition 3. Let $\mathcal{B}_{b}$ be the border of a discrete object and $p$ a point in $\mathcal{B}_{b}$. Let $T$ be a scan process of the neighboring points of $p$ in $\mathcal{B}_{b}$ and $\nu$ the greatest real value allowed. We call width- $\nu$ patch centered at $p$, and denote by $\Gamma_{\nu}(p), a$ width- $\nu$ blurred piece of discrete plane incrementally recognized from $p$ by adding points of $\mathcal{B}_{b}$ following the scan process $T$.

About the Incremental Recognition: We construct a width- $\nu$ patch centered at $p$ using the incremental recognition algorithm of blurred pieces of discrete planes introduced in [11. We add the points following the scan process $T$ and as soon as the width of the blurred piece of discrete plane becomes greater than $\nu$ we stop the recognition process.

About the Scan Process T: To uniformly spread the patch in all directions the best solution would be to scan the neighborhood of $p$ according to a geodesic distance. Nevertheless, for efficiency, we have chosen to scan this neighborhood according to a chamfer mask $\langle 3,4,5\rangle$ which is a good approximation of the geodesic distance 1415. The aim is to have a well-balanced patch around $p$ which looks almost circular. To implement this behaviour we use a priority queue $Q$. This method has originaly been proposed by Verwer et al. [16] and first used by Coeurjolly on digital surfaces [17. We start by pushing $p$ into the queue with a weight equals to zero. Then, while we do not exceed the limit width $\nu$, we pop out of $Q$ the point $v$ with the lowest weight $w$ and we add $v$ to the blurred 
piece of discrete plane we are recognizing. We then add the border voxels of the 26-neighborhood of $v$ into the priority queue as follows:

- the 6-neighbors are added in $Q$ with a weight of $w+3$,

- the strict 18-neighbors are added in $Q$ with a weight of $w+4$,

- and finally the strict 26-neighbors are added in $Q$ with a weight of $w+5$.

To stay homeomorphic to a topological disk, we take care to add a point only if it does not create a hole in the patch, by using the technique described in [18]. With this method we obtain patches like those in Fig. 2.

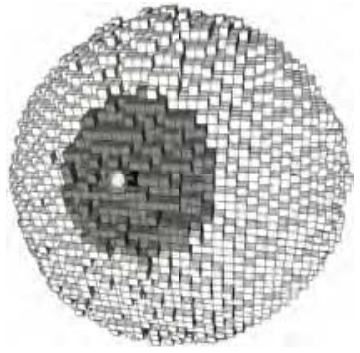

(a)

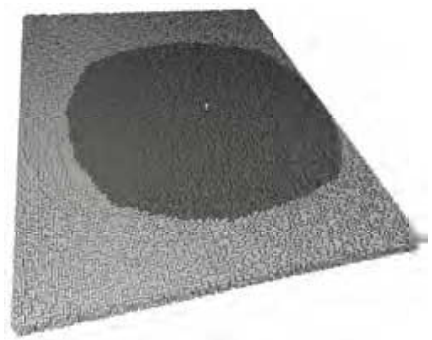

(b)

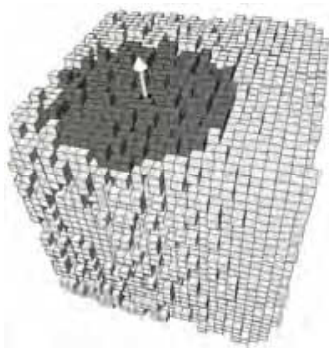

(c)

Fig. 2. An example of width-2 patches spread on the surface of different noisy objects. (a) A sphere of radius 20, (b) a $150 \times 6 \times 200$ box and (c) a cube of edge 25 .

\section{Patch Features}

\subsection{Intuition}

A patch $\Gamma_{\nu}(p)$, as previously defined, characterizes the planarity of the surface around $p$ (with respect to the width $\nu$ ). Thus, the more the patch is spread, the lesser the surface around $p$ is bent.

In addition, if the growth of $\Gamma_{\nu}(p)$ stopped, it means that the close neighboring points outside $\Gamma_{\nu}(p)$ would bend the patch too much if they were added. In that case the patch could no longer be regarded as flat. Therefore, it is possible to deduce a conformation of the discrete surface around $p$ by studying the patches centered along the points of the outline of $\Gamma_{\nu}(p)$.

To quantify these observations we have to compute the patch area, extract the outline of $\Gamma_{\nu}(p)$ and study the lie of the patches in the neighborhood of $\Gamma_{\nu}(p)$. This is what we present in the following sections.

\subsection{Width- $\nu$ Normal}

With the previous intuition we can see that the normal vector of $\Gamma_{\nu}(p)$ is a good estimation of the normal at $p$. Thus, assimilating the normal vector of $\Gamma_{\nu}(p)$ to the normal of the surface at $p$, we define a normal vector estimator for each point of the surface of a possibly noisy discrete object. 
Definition 4. Let $\mathcal{B}_{b}$ be the border of a discrete object and $p$ a point of $\mathcal{B}_{b}$. We call width- $\nu$ normal at $p$ the normal vector

$$
\overrightarrow{n_{\nu}}(p)=\vec{n}\left(\Gamma_{\nu}(p)\right)
$$

where $\vec{n}\left(\Gamma_{\nu}(p)\right)$ is the normal vector of the patch $\Gamma_{\nu}(p)$.

Other normal estimators have been proposed before. For instance, L. Papier 4 estimates the normal vector at a point $p$ of the surface using a weighted mean of the elementary unit normals of the surfels in the neighborhood of $p$ (umbrella of order $n$ ). The size of the neighborhood around $p$ can be set with the parameter $n$. In [13, A. Lenoir uses the slices of the object along the three canonical planes. He first computes tangential lines through $p$ in these slices using partial derivatives computation (implemented by convolution products). He then gives a normal vector estimation using these tangential lines. The size of the neighborhood around $p$ can be set with a scale factor. Although these methods can deal with noise, the considered neighborhood is not adaptive, contrary to our approach which fits the shape of the surface. Some results on noisy and

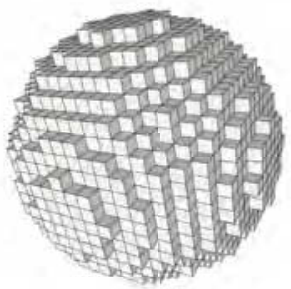

(a)

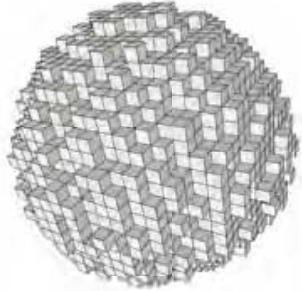

(b)

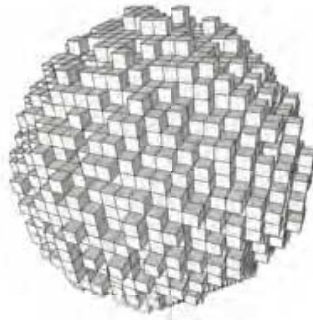

(c)

Fig. 3. A discrete sphere of radius 10 (a) without noise, (b) weakly noisy and (c) strongly noisy

non-noisy spheres (see Fig. 3) of different radius are presented in Tab. 11. To synthetize noisy spheres we start to generate a discrete sphere (i.e. the set of points $\left\{(x, y, z) \in \mathbb{Z}^{3} \mid x^{2}+y^{2}+z^{2}<r(r+1)\right\}$ where $r$ is the radius of the sphere). We then add noise by randomly moving outward a border voxel of the sphere. To add more noise we just repeat the last step.

We have compared the estimated normal vectors to the theoretical normal vector: 2 at $p$. The mean angular value $\delta_{m o y}$, in degrees, the maximal angular value $\delta_{\max }$ and the standard deviation $\sigma$ are presented in Tab 1 .

We can see that with our method the greater the radius of the spheres is, the better the estimation is, contrary to the method based on the 26-neighborhood where there is no real improvement. It is due to the fact that our method is adaptive and considers a wider neighborhood when the sphere radius increases.

${ }^{2}$ The theoretical normal vector is the vector starting from the center of the sphere to $p$. 
Table 1. Comparison between the proposed normal vector estimator and the one using only the 26-neighborhood. Angle values $\delta$ are in degrees ${ }^{\circ}$.

\begin{tabular}{|c|c|c|c|c|c|c|c|c|c|c|c|}
\hline \multirow{2}{*}{\multicolumn{3}{|c|}{$\frac{\text { Tested spheres }}{\text { Radius }}$}} & \multicolumn{3}{|c|}{ Without noise } & \multicolumn{3}{|c|}{ Weakly noisy } & \multicolumn{3}{|c|}{ Strongly noisy } \\
\hline & & & 10 & 20 & 40 & 10 & 20 & 40 & 10 & 20 & 40 \\
\hline \multirow{9}{*}{ Width } & \multirow{3}{*}{$\nu=1$} & $\delta_{\text {moy }}$ & 3.66 & 2.05 & 1.04 & 10.36 & 9.08 & 9.04 & 15.30 & 14.10 & 14.33 \\
\hline & & $\delta_{\max }$ & 8.21 & 6.10 & 3.25 & 52.53 & 91.25 & 101.24 & 95.46 & 101.42 & 117.80 \\
\hline & & $\sigma$ & 1.83 & 1.07 & 0.58 & 7.70 & 7.50 & 7.82 & 12.46 & 11.90 & 11.95 \\
\hline & \multirow{3}{*}{$\nu=2$} & $\delta_{\text {moy }}$ & 2.33 & 1.25 & 0.77 & 3.66 & 2.39 & 1.46 & 5.65 & 4.57 & 4.35 \\
\hline & & $\delta_{\max }$ & 9.92 & 3.35 & 2.94 & 16.86 & 10.16 & 5.83 & 46.98 & 40.54 & 52.57 \\
\hline & & $\sigma$ & 1.49 & 0.61 & 0.44 & 2.19 & 1.35 & 0.78 & 3.77 & 3.33 & 3.97 \\
\hline & \multirow{3}{*}{$\nu=3$} & $\delta_{\text {moy }}$ & 1.85 & 1.17 & 0.70 & 2.75 & 1.74 & 1.02 & 3.29 & 2.37 & 1.70 \\
\hline & & $\delta_{\max }$ & 6.49 & 3.93 & 2.58 & 8.90 & 7.46 & 3.89 & 14.63 & 8.80 & 7.32 \\
\hline & & $\sigma$ & 0.98 & 0.69 & 0.40 & 1.54 & 0.92 & 0.55 & 1.90 & 1.30 & 0.93 \\
\hline \multirow{3}{*}{\multicolumn{2}{|c|}{ 26-neighborhood }} & $\delta_{\text {moy }}$ & 4.61 & 4.50 & 4.22 & 7.55 & 7.50 & 7.51 & 8.83 & 8.69 & 8.57 \\
\hline & & $\delta_{\max }$ & 9.45 & 15.34 & 17.19 & 28.35 & 25.34 & 31.14 & 24.29 & 37.15 & 33.80 \\
\hline & & $\sigma$ & 2.70 & 2.75 & 2.81 & 4.19 & 4.10 & 4.19 & 4.52 & 4.87 & 4.80 \\
\hline
\end{tabular}

It behaves like this because when the sphere radius increases the surface around $p$ tends to be flat. For a given width the patch can thus spread more around $p$.

Moreover, when the noise level increases the estimation of the normal vector is less accurate. The bad results obtained when $\nu=1$ can be attributed to the tiny size of patches which do not always cover the 26-neighborhood of the point $p$. But when the width of the patches increases, say $\nu=2$ or 3 , the results are better than those obtained with the 26-neighborhood approach, and it is the case for noisy spheres as well as for non-noisy ones.

\subsection{Width- $\nu$ Patch Area}

Here we use the notations from [6]. Let $\mathcal{S}$ be an Euclidean surface and $\{\vec{n}\}$ its normal vector field. We can compute the area of $\mathcal{S}$ in the continuous space with the formula:

$$
\mathcal{A}(\mathcal{S})=\int_{\mathcal{S}} \vec{n}(s) d s
$$

Now, if we consider a digitization $D(\mathcal{S})$ of $\mathcal{S}$, we can replace the integral over $\mathcal{S}$ by a finite sum over the surfels $s$ of $D(\mathcal{S})$, the vector $\vec{n}(s)$ by an estimation of the normal vector at $s$ and $d s$ by a dot product with the unit orthogonal vector of $s$ pointing outward. The idea is to compute the contribution of each surfel to the global area of $D(\mathcal{S})$ by projecting the surfel according to the direction of the normal vector. The discrete version of the previous equation is:

$$
E_{\mathcal{A}}(D(\mathcal{S}))=\sum_{s \in D(\mathcal{S})} \vec{n}^{*}(s) \cdot \vec{n}_{e l}(s)
$$




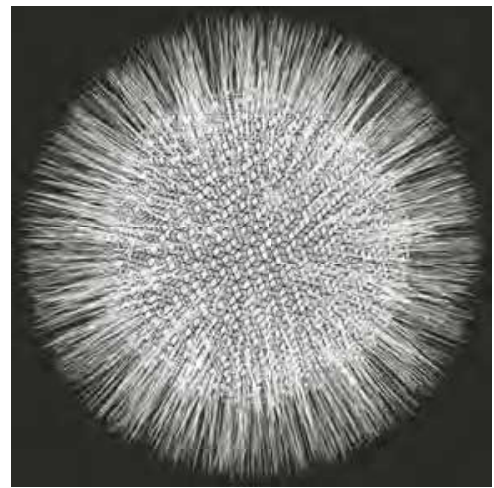

(a)

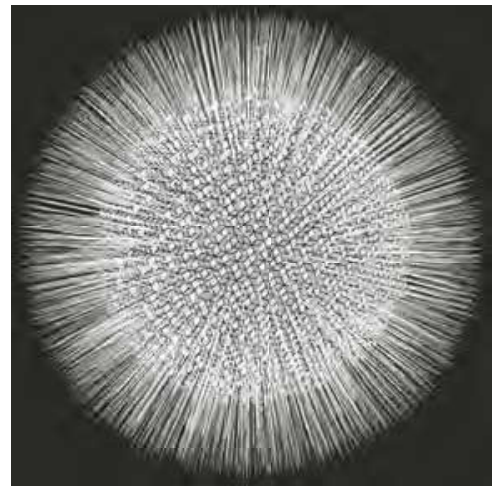

(b)

Fig. 4. Normal vector estimation on a weakly noisy sphere of radius 20 (a) using only the 26-neighborhood and (b) using the patch based approach with $\nu=3$

where $\vec{n}^{*}(s)$ is an estimation of the normal vector associated to the surface element $s$ in $D(\mathcal{S})$ and $\vec{n}_{e l}(s)$ is the elementary normal vector of $s$.

This method was first proposed in $3 \mathrm{D}$ by A. Lenoir in [13. Then, in [6], Coeurjolly et al. proved that this estimator is convergent if and only if the normal vector estimation is convergent.

In our case we can use the normal estimator proposed in section 3.2 with the previous formula to obtain an estimation of the area for the surface of a width- $\nu$ patch:

$$
E_{\mathcal{A}}\left(\Gamma_{\nu}(p)\right)=\sum_{s \in \mathcal{S}_{\Gamma_{\nu}(p)}} \vec{n}_{\nu}(p) \cdot \vec{n}_{e l}(s)={\overrightarrow{n_{\nu}}}(p) . \sum_{s \in \mathcal{S}_{\Gamma_{\nu}(p)}} \vec{n}_{e l}(s)
$$

where $\mathcal{S}_{\Gamma_{\nu}(p)}$ denotes the set of surface surfels of $\Gamma_{\nu}(p)$.

\subsection{Patch Outline}

To study the surrounding patches of $\Gamma_{\nu}(p)$, we have to clarify the notion of surrounding. We have chosen to study the conformation of the patches which are centered on points belonging to the outline of $\Gamma_{\nu}(p)$.

Let $\mathcal{B}_{b}$ be the border of a discrete object $\mathcal{O}_{b}$. We denote by $\mathcal{S}_{b}$ the set of surfels of $\mathcal{B}_{b}$ which are incident to a point that does not belong to $\mathcal{O}_{b}$, and $\mathcal{S}_{\Gamma_{\nu}(p)}$ the subset of $\mathcal{S}_{b}$ that belongs to $\Gamma_{\nu}(p)$. A point $q$ belongs to the outline of $\Gamma_{\nu}(p)$ if the voxel representation of $q$ has a surfel $s \in \mathcal{S}_{\Gamma_{\nu}(p)}$ and if there exists a surfel $s^{\prime} \in \mathcal{S}_{b} \backslash \mathcal{S}_{\Gamma_{\nu}(p)}$ such that $s$ and $s^{\prime}$ are adjacent by edge. An example of the outline of a patch is shown in Fig. 5 . 


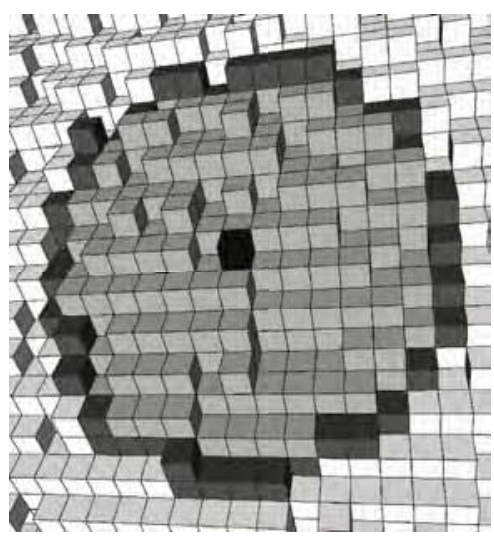

Fig. 5. The outline (in dark grey) of a width-2 grey patch centered at the black point

\section{Shape}

\subsection{Concave and Convex Parts}

With the different notions defined in the previous sections, we present an estimator that permits to characterize the shape (concave, convex or flat) around a border point of a discrete object.

The idea is to start by spreading a patch $\Gamma_{\nu}(p)$ and to compute its outline $C$. For each point $q_{i, 1 \leq i \leq|C|}$ in $C$, we then develop the neighboring patches $\Gamma_{\nu}\left(q_{i}\right)$. From these patches we obtain a vector field $\left\{\overrightarrow{n_{\nu}}\left(q_{i}\right)\right\}$ on the outline $C$. This vector field is shown in Fig. 6 for a convex and a concave area.

Observation: In the concave parts the vector field $\left\{\overrightarrow{n_{\nu}}\left(q_{i}\right)\right\}$ points towards the normal vector $\overrightarrow{n_{\nu}}(p)$ of the central patch, but in the convex parts the vector field has the reverse tendency. It is thus possible to distinguish concave and convex parts with the value of the oriented angle between the normal vector $\overrightarrow{n_{\nu}}(p)$ and the vectors $\left\{\overrightarrow{n_{\nu}}\left(q_{i}\right)\right\}$. Our estimator is based on this observation. Let $C$ be the set of points that belong to the outline of $\Gamma_{\nu}(p)$. Our shape estimator of the surface around a point $p$ is then given by the formula :

$$
\mathcal{F}_{\nu}(p)=\frac{1}{|C|} \sum_{\forall q \in C}\left(\overrightarrow{n_{\nu}} \widehat{(p), \overrightarrow{n_{\nu}}}(q)\right) \cdot \frac{E_{\mathcal{A}}\left(\Gamma_{\nu}(q)\right)}{E_{\mathcal{A}}\left(\Gamma_{\nu}(p)\right)}
$$

where $\left(\overrightarrow{n_{\nu}} \widehat{(p), \overrightarrow{n_{\nu}}}(q)\right)$ is the oriented angle value between the two normal vectors. So, the estimator $\mathcal{F}_{\nu}(p)$ is a weighted mean of the angle values between $\overrightarrow{n_{\nu}}(p)$ and the $\overrightarrow{n_{\nu}}\left(q_{i}\right)_{1 \leq i \leq|C|}$.

Angle Orientation: We determine the sign of the angle value $\left(\overrightarrow{n_{\nu}}\left(\overrightarrow{p), \overrightarrow{n_{\nu}}}(q)\right)\right.$ as shown in Fig 7. To implement this process, we compute the cross products 


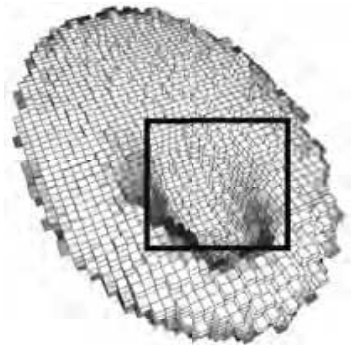

(a)

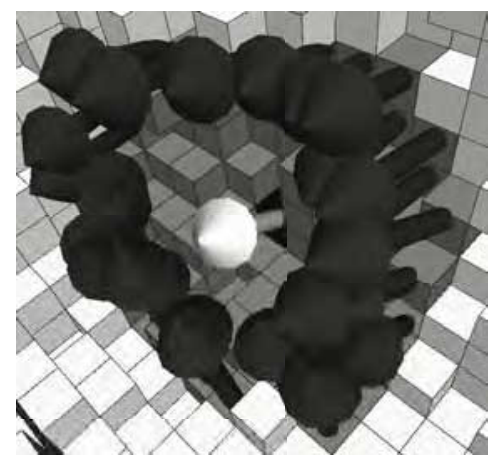

(c)

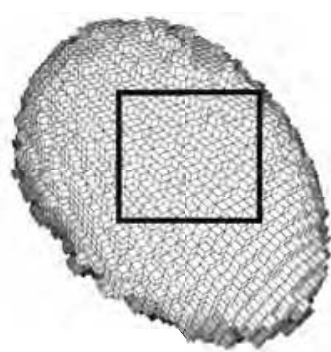

(b)

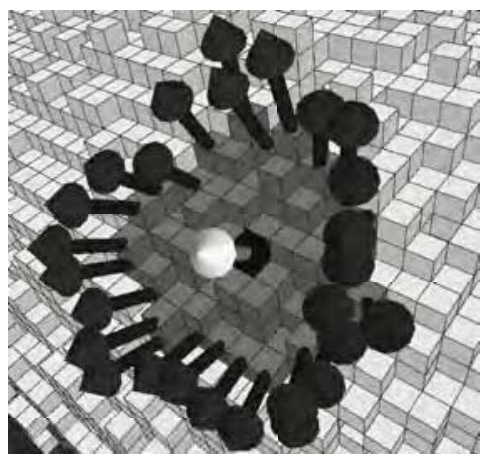

(d)

Fig. 6. Vectors layout on the outline of a patch $(a, c)$ in a convex area and $(b, d)$ in a concave area

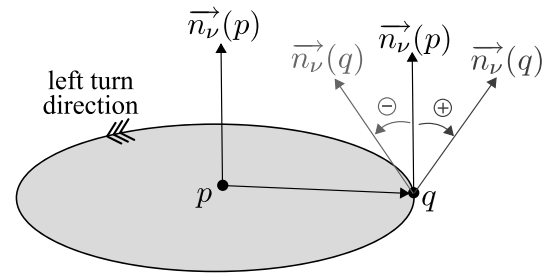

Fig. 7. Angle orientation

$\overrightarrow{n_{\nu}}(p) \wedge \overrightarrow{p q}$ and $\overrightarrow{n_{\nu}}(p) \wedge \overrightarrow{n_{\nu}}(q)$. If the two resulting vectors more or less point towards the same direction (i.e. if the scalar product $\left(\overrightarrow{n_{\nu}}(p) \wedge \overrightarrow{p q}\right) \cdot\left(\overrightarrow{n_{\nu}}(p) \wedge \overrightarrow{n_{\nu}}(q)\right)$ is positif) then the angle value is counted positively, otherwise the angle value is counted negatively. The computation of the previous cross products are feasible only if the vectors are not collinear. And the scalar product also does not have to be nil. Usually, these conditions are always verified. But sometimes, especially when the patch is very small, some degenerate cases can occur. Because they do not bring a useful shape information we can easily get rid of these cases by ignoring them and going on with the next point of the outline. 
Weighting Factor: It is clear that a wide patch $\Gamma_{\nu}(p)$ means that the surface around $p$ tends to be flat (according to the width $\nu$ ). Thus, the wider $\Gamma_{\nu}(p)$ is, the lesser the influence of the surrounding patches is to consider. Hence, the weighting factor $\frac{1}{E_{\mathcal{A}}\left(\Gamma_{\nu}(p)\right)}$ is used in $\mathcal{F}_{\nu}(p)$.

On the other hand, if a neighboring patch $\Gamma_{\nu}\left(q_{i}\right)$ is wider than another neighboring patch $\Gamma_{\nu}\left(q_{j}\right)$, then it means that the surface around $q_{i}$ is a little more stable than the surface around $q_{j}$. Thus we choose to lend more influence to information given by bigger patches. Therefore, we associate the weighting factor $E_{\mathcal{A}}\left(\Gamma_{\nu}(q)\right)$ to the angle value $\left(\overrightarrow{n_{\nu}} \widehat{(p), \overrightarrow{n_{\nu}}}(q)\right)$ in the formula $\mathcal{F}_{\nu}(p)$.

Interpretation: As a result, $\mathcal{F}(p)$ is positive when the surface around $p$ is rather convex and $\mathcal{F}(p)$ is negative when the surface around $p$ is rather concave. An increasing value of $|\mathcal{F}(p)|$ means that the surface around $p$ is more strongly concave or convex. If $\Gamma_{\nu}(p)$ is big, a value $\mathcal{F}(p)$ close to 0 means that the area around $p$ is almost flat (according to the width $\nu$ we chose). If $\Gamma_{\nu}(p)$ is small,

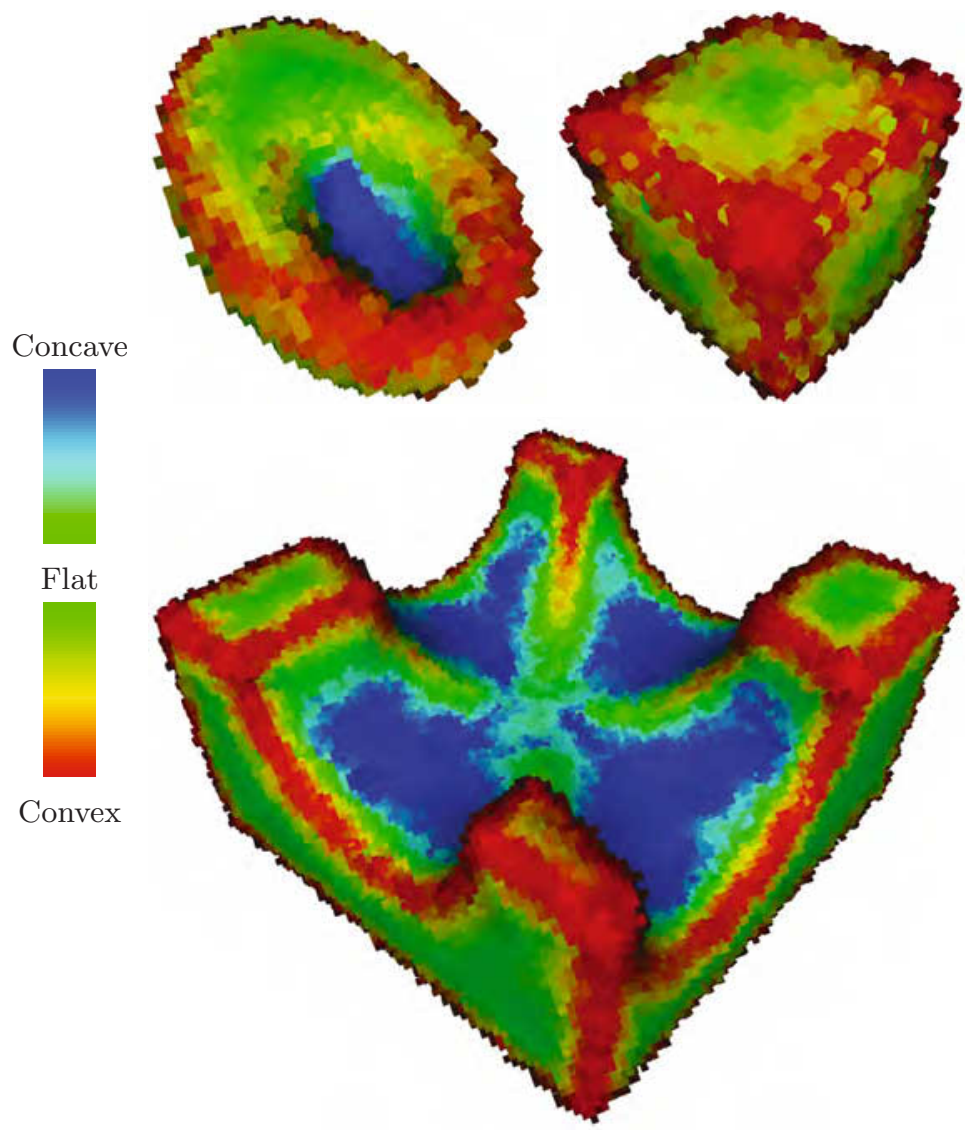

Fig. 8. Concave, convex and flat areas (according to the color scale on the left) on different objects using width-3 patches 
then the border around $p$ is strongly distorted, but in a way we can neither qualify concave, nor qualify convex (a saddle point for instance).

Some results obtained with this estimator are given in Fig. 8. To render the object we have associated to each face of a voxel $v$ the normal $\overrightarrow{n_{\nu}}(v)$.

\section{Conclusion}

In this paper we have introduced a new notion related to a point on a discrete surface : the width- $\nu$ patch centered at $p$. From this patch we have designed a robust normal vector estimator at the surface of a possibly noisy discrete object. Moreover, based on the computation of the patch area, we have proposed an estimator that characterizes the shape (concave, convex or flat) of the surface around the point $p$.

Although the approaches presented in this paper give plausible results, more formal studies on the notion of noise have to be led. A work on the convergence of the estimators could then be envisaged.

In addition more comparative studies with other methods have to be done to confirm the reliability of these estimators. In a theoretical point of view it would be interesting to see the relation of our shape estimator with the classical notion of surface curvature.

\section{References}

1. Yagel, R., Cohen, D., Kaufman, A.E.: Normal estimation in 3d discrete space. The Visual Computer 8(5\&6), 278-291 (1992)

2. Thürmer, G., Wüthrich, C.A.: Normal computation for discrete surfaces in $3 \mathrm{~d}$ space. Computer Graphics Forum 16(3), 15-26 (1997)

3. Lenoir, A.: Fast estimation of mean curvature on the surface of a $3 \mathrm{~d}$ discrete object. In: Ahronovitz, E. (ed.) DGCI 1997. LNCS, vol. 1347, pp. 175-186. Springer, Heidelberg (1997)

4. Papier, L., Françon, J.: Évaluation de la normale au bord d'un objet discret 3d. Revue internationale de CFAO et d'Infographie 13(2), 205-226 (1998)

5. Tellier, P., Debled-Rennesson, I.: 3d discrete normal vectors. In: Bertrand, G., Couprie, M., Perroton, L. (eds.) DGCI 1999. LNCS, vol. 1568, pp. 447-457. Springer, Heidelberg (1999)

6. Coeurjolly, D., Flin, F., Teytaud, O., Tougne, L.: Multigrid Convergence and Surface Area Estimation. In: Asano, T., Klette, R., Ronse, C. (eds.) Geometry, Morphology, and Computational Imaging. LNCS, vol. 2616, pp. 101-119. Springer, Heidelberg (2003)

7. Hermann, S., Klette, R.: Multigrid analysis of curvature estimators. In: Proceedings of Image and Vision Computing New Zealand, pp. 108-112 (2003)

8. Veelaert, P.: Uncertain geometry in computer vision. In: Andrès, É., Damiand, G., Lienhardt, P. (eds.) DGCI 2005. LNCS, vol. 3429, pp. 359-370. Springer, Heidelberg (2005)

9. Debled-Rennesson, I., Rémy, J.L., Rouyer-Degli, J.: Linear segmentation of discrete curves into fuzzy segments. Discrete Applied Math. 151, 122-137 (2005) 
10. Debled-Rennesson, I., Feschet, F., Rouyer-Degli, J.: Optimal blurred segments decomposition of noisy shapes in linear time. Computers \& Graphics 30(1), 30-36 (2006)

11. Provot, L., Buzer, L., Debled-Rennesson, I.: Recognition of blurred pieces of discrete planes. In: Kuba, A., Nyúl, L.G., Palágyi, K. (eds.) DGCI 2006. LNCS, vol. 4245, pp. 65-76. Springer, Heidelberg (2006)

12. Nguyen, T.P., Debled-Rennesson, I.: Curvature estimation in noisy curves. In: Kropatsch, W.G., Kampel, M., Hanbury, A. (eds.) CAIP 2007. LNCS, vol. 4673, pp. 474-481. Springer, Heidelberg (2007)

13. Lenoir, A., Malgouyres, R., Revenu, M.: Fast computation of the normal vector field of the surface of a 3-d discrete object. In: Miguet, S., Ubéda, S., Montanvert, A. (eds.) DGCI 1996. LNCS, vol. 1176, pp. 101-112. Springer, Heidelberg (1996)

14. Thiel, E., Montanvert, A.: Chamfer masks: discrete distance functions, geometrical properties and optimization. In: 11th ICPR, The Hague, The Netherlands, vol. 3, pp. 244-247 (1992)

15. Borgefors, G.: On digital distance transforms in three dimensions. Computer Vision and Image Understanding 64(3), 368-376 (1996)

16. Verwer, B., Verbeek, P., Dekker, S.: An efficient uniform cost algorithm applied to distance transforms. IEEE Transactions on Pattern Analysis and Machine Intelligence 11(4), 425-429 (1989)

17. Coeurjolly, D.: Visibility in discrete geometry: An application to discrete geodesic paths. In: Braquelaire, A., Lachaud, J.-O., Vialard, A. (eds.) DGCI 2002. LNCS, vol. 2301, pp. 326-337. Springer, Heidelberg (2002)

18. Provot, L., Debled-Rennesson, I.: Segmentation of noisy discrete surfaces. In: 12th International Workshop on Combinatorial Image Analysis, Buffalo, NY, USA, vol. 4958, pp. 160-171 (2008) 